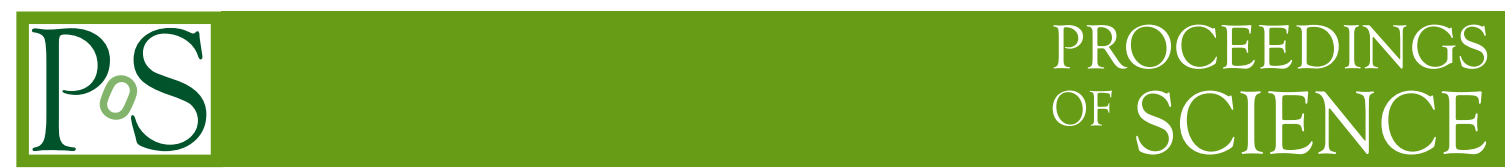

\title{
Renormalization group and bound states
}

\author{
Stanisław D. GŁAZEK* \\ University of Warsaw, Poland \\ E-mail: Stanislaw.Glazek@fuw.edu.pl
}

Similarity renormalization group procedure identifies the role of bound states in the low-energy rate of change of effective coupling constant in a model Hamiltonian with asymptotic freedom.

LIGHT CONE 2008 Relativistic Nuclear and Particle Physics

July 7-11, 2008

Mulhouse, France

${ }^{*}$ Speaker. 


\section{Introduction}

The fact that an asymptotically free interaction blows up at low energies is often associated with the concept of confinement. It is therefore of interest to construct a soluble model that exhibits a similar variation of a coupling constant and see what mechanism causes that the coupling constant rises at low energies.

In a very simple model with asymptotic freedom and a bound state, similarity renormalization group (SRG) procedure for Hamiltonians produces a strong effective interaction at low energies. The interaction strength is related to the bound-state formation and a degree to which the interactions that are responsible for the binding are included in the generator of SRG transformations: the more interaction in the generator the less increase in the coupling constant [1]. Similar results are found in the case with limit cycle, of which the apparent asymptotically free SRG behavior of effective interactions may be a part. This lecture concerns only some aspects of these results. Readers interested in a bit broader picture may consult Ref. [2]. A connection with AdS/CFT correspondence $[3,4]$ that inspires a new generation of models for calculating masses of bound states of asymptotically free quarks and gluons $[5,6]$, is not yet established.

\section{Model}

Consider a model Hamiltonian in the generic form of

$$
H=H_{0}+H_{I} .
$$

$H_{0}$ can be just a free (kinetic) energy or include also dynamical effects that are well understood. The origin of division of $H$ into $H_{0}$ and $H_{I}$ is that all eigenvalues and eigenstates of $H_{0}$ are known. Thus, $H_{0}$ provides a basis for studying new effects due to the postulated $H_{I}$. For simplicity of the model, suppose the eigenvalue problem for $H_{0}$,

$$
H_{0}|n\rangle=E_{n}|n\rangle,
$$

is solved with a set of discrete eigenvalues $E_{n}>0$ that form a monotonic sequence, $E_{m}>E_{n}$ when $m>n$, there is no degeneracy, and the corresponding eigenvectors are normalized, $\langle m \mid n\rangle=\delta_{m n}$. The interaction Hamiltonian $H_{I}$ is then defined by its matrix elements $H_{I m n}=\left\langle m\left|H_{I}\right| n\right\rangle$. An analytically soluble model to be discussed here, is obtained by assuming that these matrix elements have a factorized form, i.e., $H_{I m n}=H_{m} H_{n} . H_{n}$ should have dimension of square root of energy and the simplest choice that does not introduce any dimensionful scale is $H_{n} \sim \sqrt{E_{n}}$. The proportionality is reduced to a dimensionless number, and one can write

$$
\left\langle m\left|H_{I}\right| n\right\rangle=-g \sqrt{E_{m}} \sqrt{E_{n}},
$$

where $g$ determines the strength of the interaction, called coupling constant in analogy with QFT. The negative sign results in existence of a bound state for sufficiently large positive $g$. By definition, the bound state corresponds to a negative eigenvalue of $H$. It will have to be clarified what the words "sufficiently large $g$ " are supposed to mean, because the model Hamiltonian produces divergences (infinities) no matter how small the number $g$ is.

In summary, the model Hamiltonian is defined by its matrix elements as follows:

$$
H_{m n}=\langle m|H| n\rangle=E_{m} \delta_{m n}-g \sqrt{E_{m}} \sqrt{E_{n}} .
$$




\section{Ultraviolet divergences}

Suppose $g$ is a very small number and one expects that eigenvalues of $H$ should be nearly equal to the eigenvalues of $H_{0}$. First-order correction to $E_{m}$ is a fraction $g$ of the energy being corrected,

$$
\Delta E_{m}^{(1)}=\left\langle m\left|H_{I}\right| m\right\rangle=-g E_{m} .
$$

The second-order correction is

$$
\Delta E_{m}^{(2)}=\sum_{k \neq m} \frac{\left|\left\langle m\left|H_{I}\right| k\right\rangle\right|^{2}}{E_{m}-E_{k}}=g^{2} E_{m} \sum_{k \neq m} \frac{E_{k}}{E_{m}-E_{k}} .
$$

Terms with $k<m$ involve $E_{k}<E_{m}$ and if $E_{k} \leq E_{m} / b^{m-k}$ with a number $b>1$, they contribute an amount that is not sensitive to the lower bound on $E_{k}$, say $b^{M}$ with a large negative integer $M$, when $M \rightarrow-\infty$. Terms with $k \gg m$ involve $E_{k} \gg E_{m}$ and thus each of them contributes -1 . If $E_{k}=b^{k-m} E_{m}$, and $b \gg 1$, the second-order correction to $E_{m}$ is proportional to the number of basis states with energies (eigenvalues of $H_{0}$ ) greater than $E_{m}$. In order to obtain a finite correction, one has to limit the number of the basis states and try to understand what happens when the limit is relaxed. In analogy with QFT, imposing such a limit is called regularization. If one assumes a cutoff of the form $k \leq N$, where $N$ is a large positive integer, the result of Eq. (3.2) for $b \gg 1$ is

$$
\Delta E_{m}^{(2)}=-g^{2}(N-m) E_{m} .
$$

The ultraviolet cutoff on energies, $\Lambda=b^{N-m_{0}} E_{m_{0}}$ means $k \leq N=m_{0}+\frac{1}{\ln b} \ln \Lambda / E_{m_{0}}$, and one says that the second-order correction is ultraviolet divergent logarithmically,

$$
\Delta E_{m}^{(2)}=-g^{2} \frac{1}{\ln b} \ln \frac{\Lambda}{E_{m}} E_{m} .
$$

The divergence results from all different energy scales contributing equally to the correction. The occurrence of divergences is not limited to perturbation theory.

With the factorized interaction, solutions to the eigenvalue problem

$$
\sum_{n=M}^{N} H_{m n} \psi_{n}=E \psi_{m},
$$

have wave functions

$$
\psi_{m}=\frac{\sqrt{E_{m}}}{E_{m}-E} g \sum_{n=M}^{N} \sqrt{E_{n}} \psi_{n},
$$

in which the eigenvalue satisfies the condition

$$
1+g \sum_{n=M}^{N} \frac{E_{n}}{E-E_{n}}=0 .
$$

The sum here resembles closely the one in Eq. (3.2) and develops the same type of divergence. If a bound state exists, with a negative eigenvalue $E=-E_{B}$, one can replace the sum over $n$ by an integral, $d n=d E_{n} /\left(E_{n} \ln b\right)$, to estimate what happens, and obtains

$$
E_{B}=\frac{\Lambda-b^{M} e^{\frac{\ln b}{g}}}{e^{\frac{\ln b}{g}}-1}
$$


In the limit of $\Lambda \rightarrow \infty$ for fixed $g$, the binding energy diverges. One can also observe that the square of the matrix $H_{I}$ with large $N$ and $M$ is equal to $\frac{g b \Lambda}{1-b} H_{I}$, which means that it diverges in the ultraviolet limit of $\Lambda \rightarrow \infty$ for fixed $g$. This means that all powers of the entire $H$ are ultraviolet divergent. In particular, the evolution operator $U(t, 0)=e^{-i H t}$ does not exist in this limit.

\section{Asymptotic freedom}

The trouble with divergences can be summarized using the model as follows. One measures transition rates between, say, two states, say $\left|m_{1}\right\rangle$ and $\left|m_{2}\right\rangle$, and discovers that these rates can be described in first-order perturbation theory by an interaction Hamiltonian with matrix elements

$$
\left[\begin{array}{l}
\left\langle m_{2}\left|H_{I}\right| m_{2}\right\rangle,\left\langle m_{2}\left|H_{I}\right| m_{1}\right\rangle \\
\left\langle m_{1}\left|H_{I}\right| m_{2}\right\rangle,\left\langle m_{1}\left|H_{I}\right| m_{1}\right\rangle
\end{array}\right]=-g^{(2)}\left[\begin{array}{ll}
E_{m_{2}}, & \sqrt{E_{m_{2}} E_{m_{1}}} \\
\sqrt{E_{m_{1}} E_{m_{2}}}, & E_{m_{1}}
\end{array}\right] .
$$

The coupling constant $g^{(2)}$ corresponds to physics of the 2 states. One is then compelled to postulate that the whole matrix of $H_{I}$ has the form given in Eq. (2.3). This step is analogous to the proposal of non-Abelian gauge theory [7]. Even if this leads to divergences, one does not want to abandon the proposed interaction since it does produce a structure in perturbation theory that fits the case of states $\left|m_{1}\right\rangle$ and $\left|m_{2}\right\rangle$ and it has an appealing symmetry. Therefore, one looks for a general way out of the problem with divergences that are produced by naive extrapolation of knowledge from a small set of matrix elements to a large set. The large set is desired when one seeks a theory of presumably large range of applicability and a lot of predictive power.

The way to proceed is to learn what happens when one begins with some large $N$ and tries to mathematically reduce $N$ to a small value near $m_{2}$. As a principle, such procedure was proposed and developed in seminal Refs. [8, 9]. It is sometimes called "integrating out high-energy degrees of freedom," which applies also in statistical mechanics [10]. In the model, one starts with the eigenvalue problem

$$
H|\psi\rangle=E|\psi\rangle, \quad|\psi\rangle=\sum_{k=M}^{N} \psi_{k}|k\rangle,
$$

and applies Gaussian elimination, beginning with $\psi_{N}$. The remaining set of equations for $\psi_{k}$ with $k \leq N-1$ corresponds to (the required algebra is merely solving one linear equation)

$$
H_{I m n}^{(N-1)}=-\left(g-\frac{g^{2} E_{N}}{E-E_{N}+g E_{N}}\right) \sqrt{E_{m} E_{n}} .
$$

$H_{I}^{(N-1)}$ has the same structure of matrix elements as $H_{I}$ but contains a new "coupling constant" (the expression in the bracket). A simplification occurs for cutoffs much larger than $|E|$, for which $E / E_{N}$ can be neglected,

$$
g^{(N-1)}=g \frac{1-E / E_{N}}{1-g-E / E_{N}} \sim \frac{g}{1-g} .
$$

Therefore, one obtains a recursion that does not depend on the eigenvalue $E$,

$$
g^{(K-1)}=\frac{g^{(K)}}{1-g^{(K)}},
$$


for as long as $\Lambda_{K}=b^{K} \gg|E|$. This is the RG recursion in the model. It is solved by

$$
g^{(K)}=\frac{g}{1-g(N-K)} .
$$

Suppose the eigenvalue problem for $H^{\left(K_{0}\right)}$ with $b^{K_{0}}=\lambda_{0}$ is small enough to solve for its spectrum precisely using computers and establish that the coupling constant $g^{\left(K_{0}\right)}$ should have some value $g_{0}$ in order to reproduce some measured eigenvalue $E_{0}$ with $\left|E_{0}\right| \ll \lambda_{0}$ (some transition amplitude could be used instead). For $E_{n}=b^{n}$, Eq. (4.6) says that

$$
g_{0}=\frac{g_{\Lambda}}{1-\frac{g_{\Lambda}}{\ln b} \ln \Lambda / \lambda_{0}}, \quad g_{\Lambda}=\frac{g_{0}}{1+\frac{g_{0}}{\ln b} \ln \Lambda / \lambda_{0}} .
$$

This means that the model is asymptotically free [11, 12]: the larger the cutoff $\Lambda$ in the initial $H$ the smaller the coupling constant $g_{\Lambda}$ in it, and the smaller value is required to describe highenergy processes in perturbation theory. Calculation of $g_{\Lambda}$ means also evaluation of the counterterm required in the initial Hamiltonian.

The increase of $g^{(K)}$ when $K$ decreases causes a major difficulty because the ratio $E / E_{K}$ is compared with $1-g^{(K)}$ in the RG recursion and the unknown eigenvalue $E$ cannot be ignored when $g^{(K)} \rightarrow 1$ no matter how much smaller $|E|$ is than $E_{K}$. This is a basic obstacle to description of bound states in theories with asymptotic freedom: the eigenvalues cannot be found in perturbation theory and one cannot easily reduce the cutoff to sufficiently low values for carrying out all kinds of interesting non-perturbative calculations using available computers. In order to control what happens in the range where $g^{(K)}$ is order 1, a different RG procedure seems appropriate.

\section{Similarity RG procedure and $g_{\lambda}$ at low energies}

In the SRG procedure [13], one proceeds according to similar principles as in the standard approach described in the previous section. One also finds counterterms and evaluates effective Hamiltonians. The new idea is that one does not "integrate out" any degrees of freedom. Instead, one changes the basis states by rotating them in the Hilbert space. The rotation is designed in such a way that it guarantees the resulting Hamiltonian matrix, $H_{\lambda}$, to have vanishing matrix elements between basis states if they differ in energy by more than $\lambda$. The design is such that one does not encounter small energy denominators even when the coupling constant increases to 1 or larger values. Of course, the SRG procedure produces the same $C T$ as the one identified in the previous section and the initial condition at $\lambda=\infty$ for the SRG evolution of $H_{\lambda}$ with $\lambda$ is the same

$$
H_{\Lambda m n}=E_{m} \delta_{m n}-g_{\Lambda} \sqrt{E_{m}} \sqrt{E_{n}} .
$$

The SRG evolution can be obtained from (prime denotes differentiation with respect to $\lambda$ )

$$
H_{\lambda}^{\prime}=\left[T_{\lambda}, H_{\lambda}\right]
$$

where $T_{\lambda}=\left[G_{\lambda}, H_{\lambda}\right], G_{\lambda}=f H_{0}+(1-f) D_{\lambda}$, and $D_{\lambda}$ denotes the diagonal part of $H_{\lambda}$. For $f=0$, one has $G_{\lambda}=D_{\lambda}$, in which the diagonal part of interactions is fully included and Eq. (5.2) is the one introduced by Wegner in condensed matter physics [14, 15]. For $f=1$, one has $G_{\lambda}=H_{0}$, in which no interaction effects are included, and Eq. (5.2) is then the one used in nuclear physics [16, 
$17,18]$. For intermediate values of $f \in[0,1], G_{\lambda}$ includes interactions to an intermediate degree, correspondingly, and one can inspect what happens in various cases.

Numerical calculations produce results that can be summarized by writing

$$
H_{\lambda m n} \sim\left[E_{m} \delta_{m n}-g_{\lambda} \sqrt{E_{m}} \sqrt{E_{n}}\right] e^{-\left(E_{m}-E_{n}\right)^{2} / \lambda^{2}} .
$$

The effective coupling constant $g_{\lambda}$ is then defined using the interaction matrix elements between the states of lowest energies, such as $\left|m_{1}\right\rangle$ and $\left|m_{2}\right\rangle$ in Eq. (4.1), in analogy with the Thomson limit in QED, say $m_{1}=M, m_{2}=M+1$, and

$$
g_{\lambda}=-H_{\lambda M M+1} / \sqrt{E_{M} E_{M+1}} .
$$

A generic example is shown in Fig. 1 , where $b=4, N=16, M=-25, g_{\lambda=\infty}=g_{\Lambda} \sim 4 / 100$, and

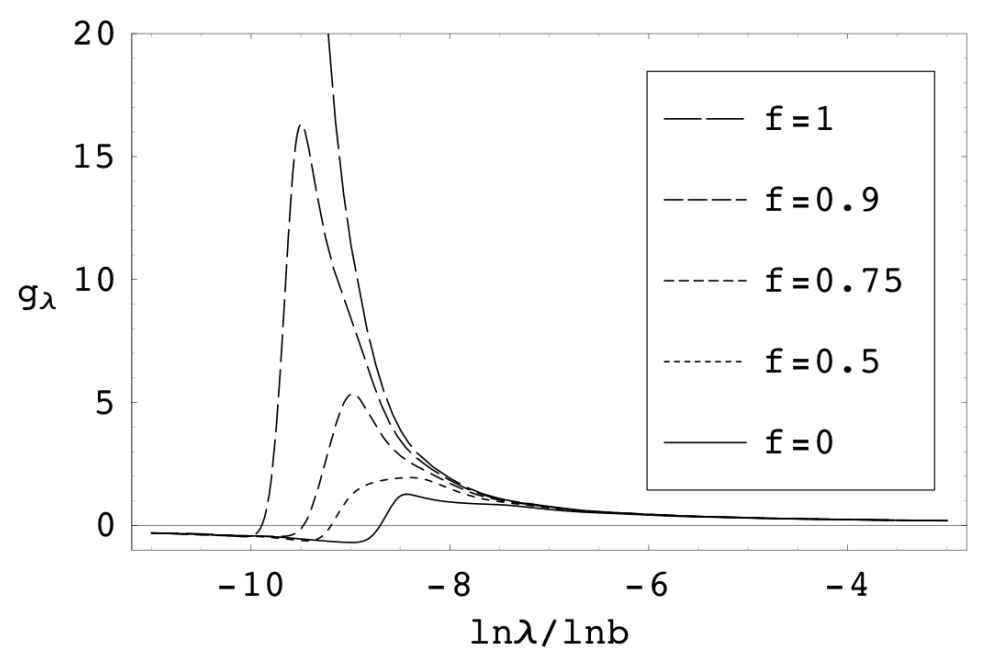

Figure 1: $g_{\lambda}$ grows toward small $\lambda$ differently for $f=0,0.5,0.75,0.9$, and 1 (the larger $f$, the higher curve). The huge increase of $g_{\lambda}$ below $\ln \lambda / \ln b \sim-8$ for $f=1$, occurs because $\lambda$ decreases there below the scale of binding energy $E_{B}$. In fact, $g_{\lambda} \rightarrow\left|E_{B}\right| / b^{M} \sim 10^{10}$. For $f=0$, the huge increase of $g_{\lambda}$ is absent.

$\Lambda=4^{16} \sim 4 \cdot 10^{9}$. The bound-state energy is $E_{B} \simeq-8 \cdot 10^{-6}$. Fig. 1 demonstrates that the increase of $g_{\lambda}$ at small energies is caused by removing interactions responsible for existence of a bound state from $G_{\lambda}$ in the generator of the SRG transformations. When the generator fully accounts for the interactions, the magnitude of the coupling constant $g_{\lambda}$ never significantly exceeds 1 .

An apparently very small alteration of $H_{I m n}$ in the model, by a term $-i h \operatorname{sgn}(m-n) \sqrt{E_{m} E_{n}}$ with a very small coupling constant $h$, leads to a new way of thinking about asymptotic freedom as a part of a limit cycle. RG limit cycles were introduced in the context of strong interactions in Ref. [19] and recently suggested relevant to the infrared behavior of QCD [20]. If $h$ is an irrational number, the model typically exhibits a chaotic RG behavior. When $h=\tan \frac{\pi}{p}$ with $p$ a large integer, a limit cycle occurs, with a period $b^{p} \sim e^{\pi / h}$. This means that $g_{\lambda_{1}}$ has the same value as $g_{\lambda_{2}}$ if $\lambda_{1}=\left(b^{p}\right)^{k} \lambda_{2}$ with integer $k$. The cycle is associated with existence of bound states whose binding energies form a geometric series with quotient $1 / b^{p}$. If a RG cycle were indeed present in a realistic extension of the standard model with some tiny coupling constant like $h$, say $\kappa$, a new generation of particle substructure would be predicted with binding energies order $e^{\pi / \kappa} \Lambda_{Q C D}$. 


\section{Conclusion}

The simple model shows that the SRG procedure may be a suitable tool to handle the increase of the coupling constant $g_{\lambda}$ in QCD when $\lambda \rightarrow \Lambda_{Q C D}$. If the generator of SRG transformations does not include interactions in $G_{\lambda}$, the effective coupling constant in the model quickly increases to very large values as soon as the SRG scale parameter $\lambda$ is lowered down to the momentum scale that characterizes formation of a bound state. This scale is much larger than the scale associated with confinement, which concerns distances far beyond the size of a single hadron. If the generator of SRG transformations includes interactions in $G_{\lambda}$, the SRG parameter $\lambda$ can be brought down to the momentum scale that characterizes the bound state and $g_{\lambda}$ does not increase to large values. The SRG procedure also helps us recognize a connection between asymptotic freedom and limit cycle, and the model example shows that in order to handle the case of limit cycle the generator of SRG transformations must include interactions in $G_{\lambda}$.

\section{Acknowledgement}

The author would like to thank Jean-François Mathiot and the Organizing Committee of LC2008 for invitation and outstanding hospitality.

\section{References}

[1] S. D. Głazek, R. J. Perry, Phys. Rev. D 78, 045011 (2008), and references therein.

[2] S. D. Głazek, in 48th Cracow School of Theoretical Physics: Aspects of Duality.

[3] J. M. Maldacena, Adv. Theor. Math. Phys. 2, 231(1998).

[4] O. Aharony et al., Phys. Rept. 323, 183 (2000).

[5] G. F. de Teramond, S. J. Brodsky, Phys. Rev. Lett. 94, 201601 (2005).

[6] S. J. Brodsky, G. F. de Teramond, Phys. Rev. Lett. 96, 201601 (2006).

[7] C.-N. Yang, R. L. Mills, Phys. Rev. 96, 191 (1954).

[8] K. G. Wilson, Phys. Rev. 140, B445 (1965).

[9] K. G. Wilson, Phys. Rev. D2, 1438 (1970).

[10] K. G. Wilson, Rev. Mod. Phys. 47, 773 (1975).

[11] D. J. Gross, F. Wilczek, Phys. Rev. Lett. 30, 1343 (1973).

[12] H. D. Politzer, Phys. Rev. Lett. 30, 1346 (1973).

[13] S. D. Głazek, K. G. Wilson, Phys. Rev D 48, 5863 (1993); 49, 4214 (1994).

[14] F. Wegner, Ann. Physik (Leipzig) 3, 77 (1994).

[15] F. Wegner, J. Phys. A: Math. Gen. 39, 8221 (2006).

[16] S. Szpigel, R. J. Perry, in Quantum Field Theory, A 20th Century Profile, Ed. A. N. Mitra, p. 59.

[17] S. K. Bogner, R. J. Furnstahl, R. J. Perry, Phys. Rev. C 75, 061001(R) (2007).

[18] S. K. Bogner, R. J. Furnstahl, R. J. Perry, A. Schwenk, Phys. Lett. B 649, 488 (2007).

[19] K. G. Wilson, Phys. Rev. D 3, 1818 (1971).

[20] E. Braaten and H.-W. Hammer, Phys. Rev. Lett. 91, 102002 (2003). 